\title{
AN APPLICATION OF YAU'S MAXIMUM PRINCIPLE TO CONFORMALLY FLAT SPACES
}

\section{S. I. GOLDBERG}

\begin{abstract}
Results of $\mathbf{M}$. Tani on compact conformally flat manifolds and of $M$. Okumura on compact hypersurfaces of Euclidean space are extended to complete spaces by an application of S.-T. Yau's "maximum principle".
\end{abstract}

1. Introduction. M. Tani [3] proved that a compact and orientable Riemannian manifold admitting a conformally flat metric of positive Ricci curvature and constant scalar curvature is a space form, that is, it is a constant curvature space. It is our purpose to extend this result to complete Riemannian manifolds with Ricci curvature bounded from below. This will be accomplished by employing a "maximum principle" due to S.-T. Yau. In fact, the following statement is obtained.

TheOREM 1. Let $M$ be $a d$-dimensional, $d>3$, complete, conformally flat Riemannian manifold whose Ricci curvature is bounded from below. If its scalar curvature $r$ is a positive constant and $\operatorname{tr} Q^{2}<r^{2} /(d-1)$, then $M$ is a space form.

2. Definitions and notation. Let $(M, g)$ be a Riemannian manifold with metric $g$. The curvature transformation $R(X, Y), X, Y \in M_{m}$, where $M_{m}$ is the tangent space at $m \in M$, and $g$ are related by

$$
R(X, Y)=\nabla_{[X, Y]}-\left[\nabla_{X}, \nabla_{Y}\right]
$$

where $\nabla$ is the Riemannian connection. In terms of a basis $X_{1}, \ldots, X_{d}$ of $M_{m}$, we set

$$
\begin{aligned}
R_{i j k h} & =g\left(R\left(X_{i}, X_{j}\right) X_{k}, X_{h}\right), \quad R_{i j}=\operatorname{tr}\left(X_{k} \rightarrow R\left(X_{i}, X_{k}\right) X_{j}\right), \\
t_{i_{1} \ldots i_{p}} & =t\left(X_{i_{1}}, \ldots, X_{i_{p}}\right), \quad \nabla_{i} t_{i_{1} \ldots i_{p}}=\left(\nabla_{X_{i}} t\right)\left(X_{i_{1}}, \ldots, X_{i_{j}}\right) .
\end{aligned}
$$

We denote the scalar curvature by $r$, that is, $r=\operatorname{tr} Q$, where $Q=\left(R_{j}^{i}\right)$ and $R_{j}^{i}=g^{i k} R_{j k}$. The manifold $(M, g)$ is conformally flat if $g$ is conformally related to a locally flat metric.

3. The Laplacian of $\operatorname{tr} Q^{2}$. The following formula may be found in [1]:

$$
\frac{1}{2} \Delta \operatorname{tr} Q^{2}=g^{a b} \nabla_{a} R^{i j} \nabla_{b} R_{i j}+R^{i j} g^{a b} \nabla_{a}\left(\nabla_{b} R_{i j}-\nabla_{i} R_{b j}\right)+\frac{1}{2} R^{i j} \nabla_{j} \nabla_{i} r+K \text {, }
$$

where $\operatorname{tr} Q^{2}$ is the square length of the Ricci tensor, and

$$
K=R^{i k}\left(R_{i}^{j} R_{j k}+R^{h j} R_{i j h k}\right) .
$$

Received by the editors July 9, 1979.

AMS (MOS) subject classifications (1970). Primary 53C20; Secondary 53A07, 53C40.

Key words and phrases. Conformally flat, Ricci curvature, scalar curvature. 
If $r$ is a constant, the third term on the r. h. s. of (3.1) vanishes. If, moreover, $M$ is conformally flat and $d>3$, the second term on the right also vanishes (see [1]) and (3.1) reduces to

$$
\frac{1}{2} \Delta \operatorname{tr} Q^{2}=K+g(\nabla Q, \nabla Q) .
$$

4. Proof of Theorem 1. Since $M$ is conformally flat it can be shown that

$$
(d-1)(d-2) K=d(d-1) \operatorname{tr} Q^{3}-r(2 d-1) \operatorname{tr} Q^{2}+r^{3} .
$$

Put $S=Q-(r / d) I, I=$ identity. Then, from $\operatorname{tr} S^{2}>0$, we see that $\operatorname{tr} Q^{2}>r^{2} / d$ with equality holding if and only if, $M$ is an Einstein space. Since $r$ is a constant, the Laplacian $\Delta f^{2}$ of the function $f^{2}=\operatorname{tr} S^{2}, f>0$, satisfies $\Delta f^{2}=\Delta \operatorname{tr} Q^{2}$. Thus,

$$
\frac{1}{2} \Delta f^{2}=K+g(\nabla Q, \nabla Q) \text {. }
$$

Moreover,

$$
(d-1)(d-2) K=d(d-1)\left(\operatorname{tr} S^{3}+\frac{3 r}{d} f^{2}+\frac{r^{3}}{d^{2}}\right)-r(2 d-1)\left(f^{2}+\frac{r^{2}}{d}\right)+r^{3}
$$

The following lemma may be found in [2].

LEMMA 1. Let $a_{i}, i=1, \ldots, d$, be real numbers with

$$
\sum_{i=1}^{d} a_{i}=0, \quad \sum_{i=1}^{d} a_{i}^{2}=k^{2}, \quad k=\text { const } \geqslant 0 .
$$

Then,

$$
-\frac{d-2}{\sqrt{d(d-1)}} k^{3}<\sum_{i=1}^{d} a_{i}^{3}<\frac{d-2}{\sqrt{d(d-1)}} k^{3} .
$$

Applying Lemma 1 to the eigenvalues of $S,(4.2)$ yields the inequality

$$
(d-1) K>f^{2}(r-\sqrt{d(d-1)} f) \text {. }
$$

We conclude from (4.1) that

$$
\frac{d-1}{2} \Delta f^{2}>f^{2}(r-\sqrt{d(d-1)} f) \text {. }
$$

LEMMA 2 (S.-T. YAU [4]). Let $M$ be a complete Riemannian manifold with Ricci curvature bounded below. Let $u$ be a $C^{2}$ function with sup $u<\infty$. Then, there exists a sequence $\left\{p_{v}\right\}$ in $M$ such that

$$
\lim _{\nu \rightarrow \infty}\left\|d u\left(p_{\nu}\right)\right\|=0, \quad \lim _{\nu \rightarrow \infty}(\Delta u)\left(p_{\nu}\right)<0, \quad \lim _{\nu \rightarrow \infty} u\left(p_{\nu}\right)=\sup u .
$$

Applying Lemma 2, the inequality (4.3) gives rise to the inequality

$$
\lim _{\nu \rightarrow \infty} f^{2}\left(p_{v}\right)\left\{r-\sqrt{d(d-1)} f\left(p_{\nu}\right)\right\}<0 .
$$

Hence, either $f^{2} \equiv 0$ or $\sup f>r / \sqrt{d(d-1)}$, the latter implying $\sup \operatorname{tr} Q^{2}>$ $r^{2} /(d-1)$. The former says that $\operatorname{tr} Q^{2}=r^{2} / d$, so $g$ is an Einstein metric. However, since $g$ is conformally flat, it is a constant curvature metric. 
The condition tr $Q^{2}<r^{2} /(d-1)$ is essential. For, if $M=M_{1} \times N$, where $M_{1}$ has constant curvature and $N$ is 1-dimensional, then $M$ is conformally flat, its Ricci curvature is bounded below, $r$ is constant and $\operatorname{tr} Q^{2}=r^{2} /(d-1)$.

In a similar manner, we obtain the following extension of a theorem of Okumura [2].

THEOREM 2. Let $M$ be a d-dimensional complete connected hypersurface of $R^{d+1}$ with Ricci curvature bounded from below. If its mean curvature tr $H$ is constant and $\operatorname{tr} H^{2}<(\operatorname{tr} H)^{2} /(d-1)$, then $M$ is a totally umbilical hypersurface.

The inequality in Theorem 2 is the best possible as one sees by considering $M=S^{d-1} \times R$.

\section{REFERENCES}

1. S. I. Goldberg, On conformally flat spaces with definite Ricci curvature, Kōdai Math. Sem. Rep. 21 (1969), 226-232.

2. M. Okumura, Hypersurfaces and a pinching problem on the second fundamental tensor, Amer. J. Math. 86 (1974), 207-213.

3. M. Tani, On a conformally flat Riemannian space with positive Ricci curvature, Tôhoku Math. J. 19 (1967), 227-231.

4. S.-T. Yau, Harmonic functions on complete Riemannian manifolds, Comm. Pure Appl. Math. 28 (1975), 201-228.

Department of MAthematics, University Of IllinoIs, Urbana, Illinois 61801 